\title{
PREDICTING THE PERFORMANCE OF TRANSACTIONAL ELECTRONIC COMMERCE PROTOCOLS
}

\author{
Matthew Berry \\ Data Network Architectures Laboratory \\ University of Cape Town, South Africa \\ chuckberry@iname.com
}

\author{
Andrew Hutchison \\ Data Network Architectures Laboratory \\ University of Cape Town, South Africa \\ hutch @cs.uct.ac.za
}

Elton Saul

Data Network Architectures Laboratory

University of Cape Town, South Africa

esaulQcs.uct.ac.za

\begin{abstract}
As security practitioners, there is a tendency to neglect performance issues. The Secure Electronic Transaction (SET) protocol is proposed as the transactional electronic commerce protocol of choice, yet it has been criticised for its complexity and denseness. This paper reports on a performance analysis of SET conducted through the development of a queueing model. The purpose of modelling SET was to predict its performance and determine which stages in the protocol are likely bottlenecks and points for optimisation when doing SET implementations. Results reveal that existing infrastructures will generally suffice for SET, financial network authorization delay directly affects performance and that transaction times are strongly dependent on the merchant and gateway processing time.
\end{abstract}

Keywords: Performance, SET, electronic commerce 


\section{INTRODUCTION}

The performance of a new protocol is important to its users. While a protocol may have all the security requirements and redundancy required, it is ultimately its usability which will determine its success or failure. The Secure Electronic Transaction (SET) protocol has been proposed as the transactional electronic commerce protocol of choice, yet some businesses are hesitant to embrace it because it is perceived as cumbersome and complex. Realistic and acceptable response times will affect the success of SET, and in this paper a multiclass queueing network model of SET is developed to predict its performance. The effect of different infrastructures is investigated using this model.

The structure of the paper is as follows: in Section 2, an overview of SET is presented; in Section 3 a motivation for performance modelling is given; Section 4 explains the multiclass queueing model used, and this is elaborated on before results are presented and conclusions drawn.

\section{THE SET PROTOCOL}

The SET protocol [1] is a payment system that was developed by a number of important role-players in the financial arena to facilitate secure payment card transactions over open networks such as the Internet. The SET specification defines a number of transaction protocols that employ cryptographic concepts to conduct secure electronic commerce. These transactions take place between six major roleplayers:

1. A cardholder possesses and uses a payment card that has been issued by an issuer of a particular brand.

2. An issuer is a financial institution that has an established account with the cardholder and issues a payment card as a token of his possession of the account.

3. A merchant offers goods for sale or provides services to the cardholder in exchange for payment. The merchant must have an established relationship with an associated brand's acquirer.

4. An acquirer is a financial institution that establishes an account with the merchant and authorizes payment for the cardholder.

5. A payment gateway is a device operated by the acquirer or a designated third party that processes merchant authorization and payment requests.

6. A certificate authority issues digital certificates to participants which allows them to be identified, verified and authenticated. 
There are certificate authorities for the cardholder, merchant and payment gateway.

The different transactions that the SET specification supports are listed below in Table 1.

\begin{tabular}{||l|l|l||}
\hline Certificate Management & Cardholder Registration & Merchant Registration \\
\cline { 2 - 3 } & Inquiry and Status & Certificate Request \\
\hline Cardholder-Merchant & Purchase Request & Purchase Inquiry \\
\hline Merchant-Payment & Payment Authorization & Authorization Reversal \\
\cline { 2 - 3 } Gateway & Payment Capture & Capture Reversal \\
\cline { 2 - 3 } & Credit Request & Credit Reversal \\
\cline { 2 - 3 } & Batch Administration & \\
\hline
\end{tabular}

Table 1 Transactions in SET protocol

In this paper the SET purchase request transaction has been modelled. This transaction was selected because it is the central and most common operation within SET.

\section{MOTIVATION FOR PERFORMANCE MODELLING}

One of the first researchers who addressed performance and optimisation in security protocols was Li Gong. In [7] lower bounds were explored and it was shown how the number of messages and rounds could be reduced. This theme is significant since besides the cryptographic overhead which security protocols incur, the message structures and construction also influence performance. It was with the intention of gaining insight into the performance of SET that we set about developing a performance model. An analytical modelling approach was chosen, and a multiclass queueing network model was constructed. In the following sections, details of the modelling process and parameters are provided.

\section{MULTICLASS QUEUING NETWORKS}

In a multiclass queueing network a queue consists of a waiting line and one or more servers. Customers, requiring resources, arrive with a certain interarrival time distribution and are served according to some service discipline (e.g. priority, FIFO). The duration of service has a particular service time distribution (see [8] for a more thorough theoretical background). A queueing network consists of a number of such queues where customers move from one queue to another with a certain probability. 


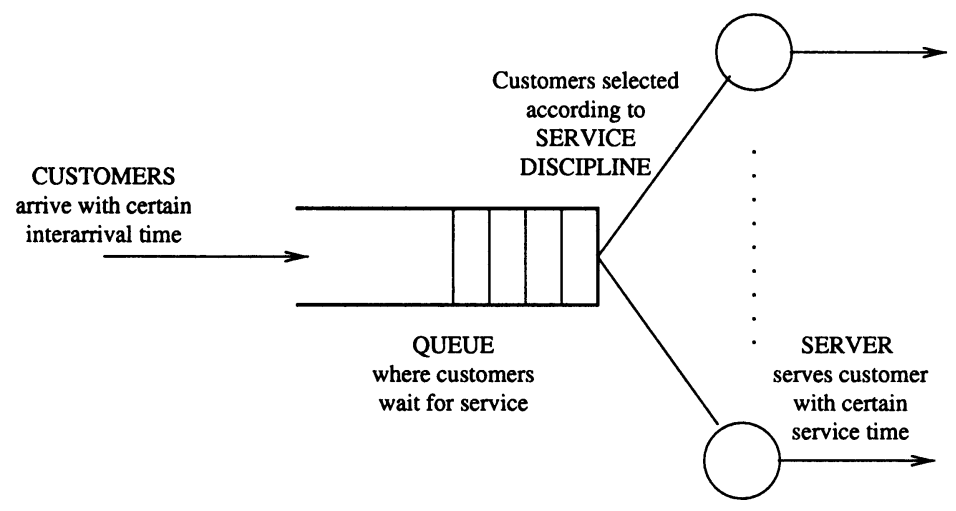

Figure 1 A Queue

A system can be modelled by a series of queues, representing the resources of the system being modelled, where the customers represent the load on each queue. Customers with similar routing behavior and service demands are grouped together as a workload. Within each workload there may exist a number of customer classes. A class is used to describe how the demand of a customer in a particular workload may change when he returns to the same queue on the next visit. Customers are allowed to change classes as they are routed through the network which can be used to account for the different workload demands of the same customer at the same queue.

A route is defined as a path that a workload follows through the network. If customers leave a particular queue and eventually return to that queue, the workload is described as a closed workload. When not closed, the workload is referred to as being open.

Each queue has a certain service discipline. This describes how the next customer in the queue is selected for service. Disciplines allowed for by the so-called product form networks [3] are infinite server (IS), lastcome-first-served (LCFS), first-in-first out (FIFO) and processor sharing (PS). In FIFO, customers receive service in order of entry into the queue. IS is equivalent to a random delay. In PS, customers also receive service immediately, but the service time is inversely proportional to the number of customers being concurrently served.

By modelling a system as a mutliclass queuing network, its average performance can be predicted. Valuable statistics, such as average queue length and utilization provide insight into the behavior of the system as a whole. 
The Stochastic Network Analysis Programming Language, SnapL [4], can be used to describe a queueing network model of a system to be analyzed for performance by MicroSnap [6].

\section{DEVELOPING THE MODEL}

Performance analysis of the SET purchase request transaction is done with a multiclass queueing network model. The basic model, shown in Figure 2, has four queues, namely Cardholder, Internet, Merchant and Gateway through which the workload is routed. Each class in the workload represents a particular message within the protocol. This allows the queues to differentiate between the different resource demands of the messages. For example, the PInitReq workload class is associated with the purchase request initiation message which is routed from the Cardholder queue to the Merchant queue.

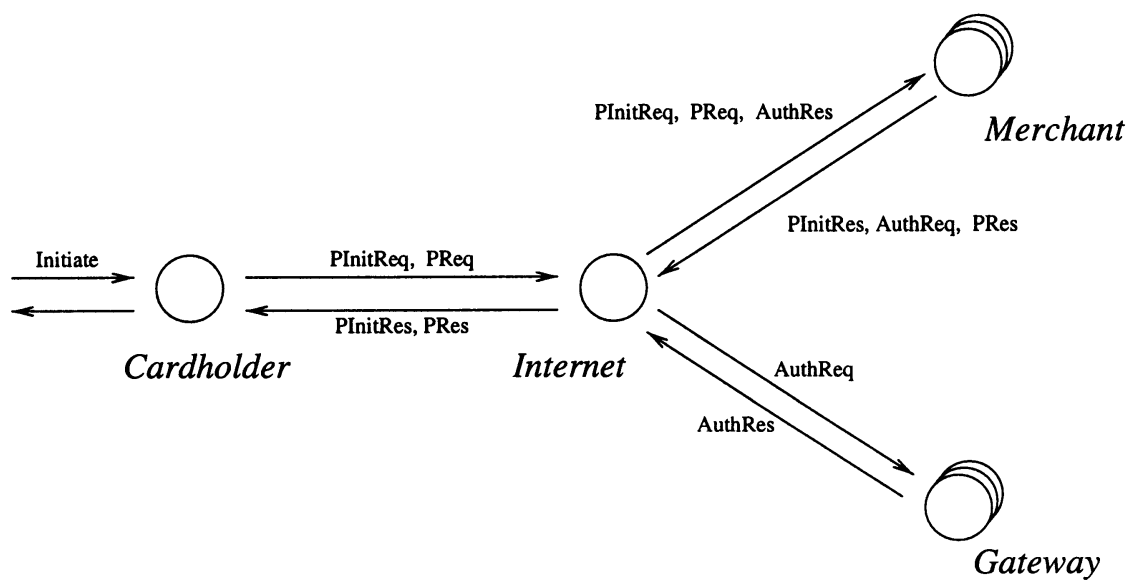

Figure 2 Multiclass queuing network for SET Purchase Request.

A request arrives at Cardholder as a customer of class Initiate. It changes class to PInitReq and is routed to Internet which, after an appropriate service time, routes PInitReq to Merchant where it changes class to PInitRes and is returned to the Internet from where it is returned to Cardholder. On leaving Cardholder, the PInitRes changes class to PReq and is routed to Internet and then Merchant. Here it changes class to AuthReq and is routed to Internet and Gateway. Leaving Gateway it changes to AuthRes, the new class it routed back to Merchant via Internet. The class then changes to PRes and routes to Internet and Cardholder. The queueing network thus has a single open workload consisting of the classes mentioned above. 
The Cardholder and Internet queues are modelled with infinite servers, while the Merchant and Gateway queues are modelled using the PS service discipline. The cardholder software takes a certain amount of time to generate the request messages for the merchant and to process the incoming responses. Although there might be simultaneous requests within the system, the generation and processing of each cardholder is independent of other such requests. The Internet queue models the time taken to deliver the message from the source end-entity to the destination end-entity. This assumption is realistic as messages will generally follow different paths and thus not influence each other. The physical capacity and latency of the link determines this delay. At the merchant and payment gateway, response times of the server decrease as the number simultaneous requests increases. The software at these entities must process transactions concurrently and thus a processor sharing model is used.

\section{MODEL PARAMETERS}

The mean service time of the Internet queue is derived from physical propagation delay associated with the channel connecting the two communicating entities. This propagation delay is a function of the channel capacity and latency (which are determined by its physical characteristics) and the size of the data packet being transmitted. Mathematically,

$$
d e l a y \_i n \_s e c s=\frac{s i z e \_i n \_b y t e s \times 8}{c a p a c i t y \_i n \_b p s}+l a t e n c y \_i n \_s e c s
$$

The size of each data packet, i.e. the size of the messages, was measured from an implementation of the SET purchase request that was written for the purpose of gathering data for this performance model [9]. Access to such realistic values allows for meaningful experimentation with this and other similar protocols. Note that these values (Table 2) are accurate to the nearest ten bytes, since the sizes of the messages are expected to vary slightly. This could be caused by varying lengths of string fields. The AuthRes and PRes could also contain brand-specific data extensions which would influence their sizes. No other realistic data are available. Since the messages are relatively small, latency can be significant and must thus be included in the model.

It is expected that most cardholders will participate in SET using a modem-link to their internet service provider. According to the National Electronic Commerce Resource Center [2], it is projected that most individuals will still be using $28.8 \mathrm{Kbps}$ modems by the year 2001 . Although modem capacities may vary, latency tests using ping and traceroute indicate that a modem's intrinsic latency is between 75 and 130 millisec- 


\begin{tabular}{||l|c||}
\hline SET Message & Size (bytes) \\
\hline PInitReq & 160 \\
\hline PInitRes & 8200 \\
\hline PReq & 2310 \\
\hline PRes & 4910 \\
\hline AuthReq & 9120 \\
\hline AuthRes & 4850 \\
\hline
\end{tabular}

Table 2 Measured Message Sizes (accurate to 10 bytes)

onds [5]. The most common server connection capacity is $10 \mathrm{Mbps}$, followed by $1 \mathrm{Mbps}$ and $56 \mathrm{Kbps}$. Latency is between 0.3 milliseconds for Ethernet and 10 milliseconds for ISDN. The model must thus take into account the different effects of the cardholder-merchant and merchantgateway links.

The service times of each of the queues is derived from time taken for the entity's software to process a request and then generate a response. Using the implementation, the values in Table 3 were measured ${ }^{1}$. For example, the service time of the Merchant queue for the PReq class is derived from the time taken for the implementation to read, decode and verify the PReq message and then generate the corresponding AuthReq message. Although these times measured from a Java implementation may be slower than other implementations, relative comparisons still produce realistic results.

Since the purchase request is also dependent on the time taken by the gateway to authorize a payment, this is included as a model parameter. This delay is directly influenced by the external financial network.

\begin{tabular}{||l|c|c||}
\hline \multicolumn{1}{||c|}{ Processing } & $\begin{array}{c}\text { Measured } \\
\text { Time (ms) }\end{array}$ & $\begin{array}{c}\text { Model } \\
\text { Value (ms) }\end{array}$ \\
\hline Cardholder generates PInitReq & $61 \pm 47$ & 60 \\
\hline Merchant reads PInitReq, generates PInitRes & $1155 \pm 412$ & 1160 \\
\hline Cardholder reads PInitRes, generates PReq & $1755 \pm 272$ & 1760 \\
\hline Merchant reads PReq, generates AuthReq & $1523 \pm 168$ & 1520 \\
\hline Gateway reads AuthReq, generates AuthRes & $2491 \pm 755$ & 2490 \\
\hline Merchant reads AuthRes, generates PRes & $1051 \pm 12$ & 1050 \\
\hline Cardholder reads PRes & $644 \pm 35$ & 640 \\
\hline
\end{tabular}

Table 3 Measured Times (with Standard Deviations) for Generating Response Messages with Actual Values used in Model. 
The effect of the financial network on the performance can thus also be measured.

The performance of the purchase request is predicted by examining the residence time of the workload. The residence time of a workload is defined as the time it spends from its instant of entry until it leaves the open network. The utilization of the merchant and gateway services will also be examined.

\section{RESULTS}

Figure 3 shows the effect of varying the customer interarrival times (IAT). The characteristics of cardholder-merchant and merchant-gateway links is kept constant. The result of varying the IAT indicates how the merchant will handle the load. If the IAT is sufficiently large, it has the effect of only one customer being served by the merchant. Thus the lower bound for the transaction time is measured.

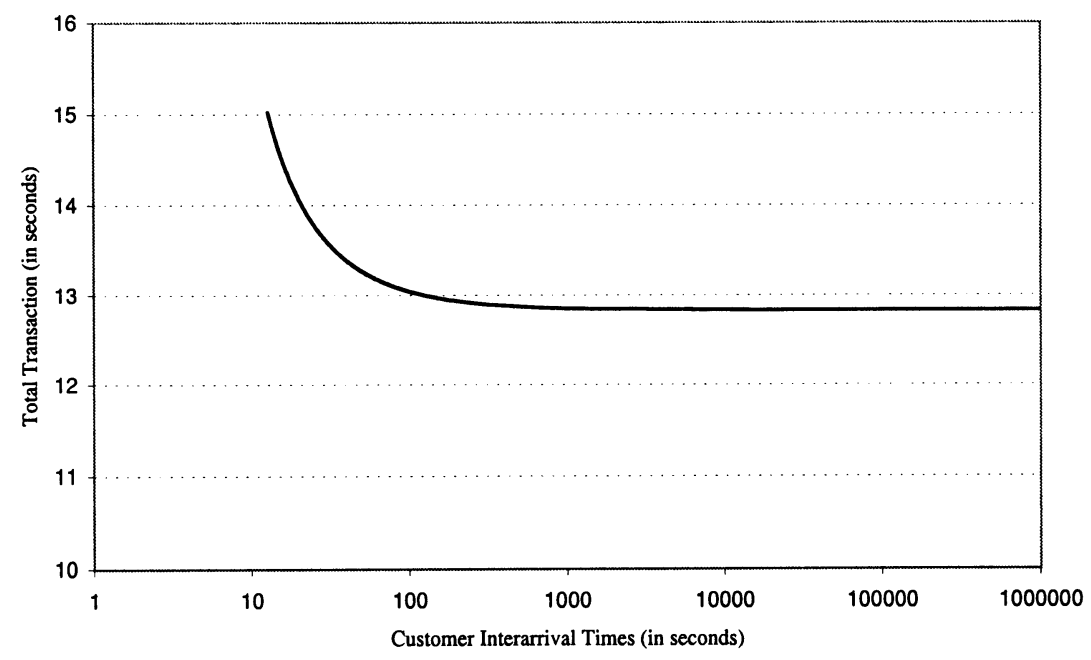

Figure 3 Graph of Total Transaction Time vs Customer Interarrival Times. The Cardholder-Merchant link is modelled as $28.8 \mathrm{Kbps}$, while the Merchant-Gateway link is $1 \mathrm{MBps}$. The financial network delay is $100 \mathrm{~ms}$.

Figure 4 shows the component times at this lower bound. Notice that a large percentage $(32.8 \%)$ of the transaction time is spent by the Merchant processing the requests. Although the gateway's contribution $(21.9 \%)$ is a lot less, this figure will increase as the number of merchants 


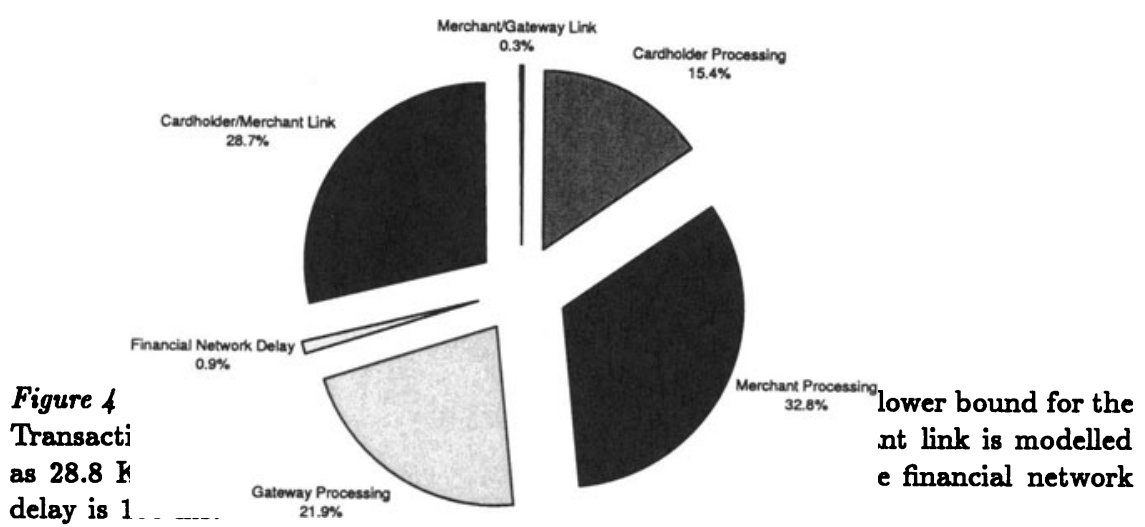

per gateway increases (this effect is measured later). It is thus important that these times are kept to a minimum. A significant time is added by the message propagation delay between the cardholder and merchant.

As the IAT is decreased, i.e. the rate of purchase requests increases, the transaction time approaches a horizontal asymptote, representing the upper bound of the request rate. Above this number, the merchant is not be able to process requests fast enough and the system becomes unstable.

The effect of the cardholder-merchant link on the transaction time was tested with the model. It is apparent from Table 5 that the link's latency effect is negligible (less than $0.6 \%$ in the worst case). As expected, the latency effect adds a constant amount to the transaction. For example, when the latency is increased by $25 \mathrm{~ms}$ from $75 \mathrm{~ms}$ to $100 \mathrm{~ms}$, the transaction time increases by $4 \times 25 \mathrm{~ms}=100 \mathrm{~ms}$ (there are four messages exchanges between the cardholder and merchant).

The results in Table 5 are plotted in Figure 5. The graph shows that using a $28.8 \mathrm{Kbps}$ modem is only $16 \%$ slower than using a $56 \mathrm{Kbps}$ modem, the current highest capacity modem on the market. It appears that bandwidths faster than $56 \mathrm{Kbps}$ will have little improvement, since the transaction time is effected more by the rest of the system. 


\begin{tabular}{||c|c|c||}
\hline Bandwidth & \multicolumn{2}{|c||}{ Latency (ms) } \\
\cline { 2 - 3 } (kbps) & 0.3 & 10 \\
\hline 56 & 14.79 & 14.81 \\
\hline 64 & 14.54 & 14.56 \\
\hline 1000 & 12.91 & 12.93 \\
\hline 10000 & 12.81 & 12.83 \\
\hline
\end{tabular}

Table \& Transaction Times (in seconds) for Different Bandwidths and Latencies of the Merchant-Gateway Link. The Cardholder-Merchant link is $28.8 \mathrm{Kbps}$ and there is a $100 \mathrm{~ms}$ financial network delay.

The effect of the merchant-gateway link was also investigated. Again, from Table 4, the latency of the link is constant and negligible. The graph of these values (see Figure 6) shows that there is little difference between the $1 \mathrm{Mbps}$ and $10 \mathrm{Mbps}$ link. Although these are ideal link capacities, lower capacities are still acceptable. The use of a $64 \mathrm{KBps}$ ISDN link is only $13.5 \%$ slower.

As expected, the delay caused by the authorization request (on the existing financial network) directly affects the total transaction time. The effect is illustrated in the graph of Figure 7.

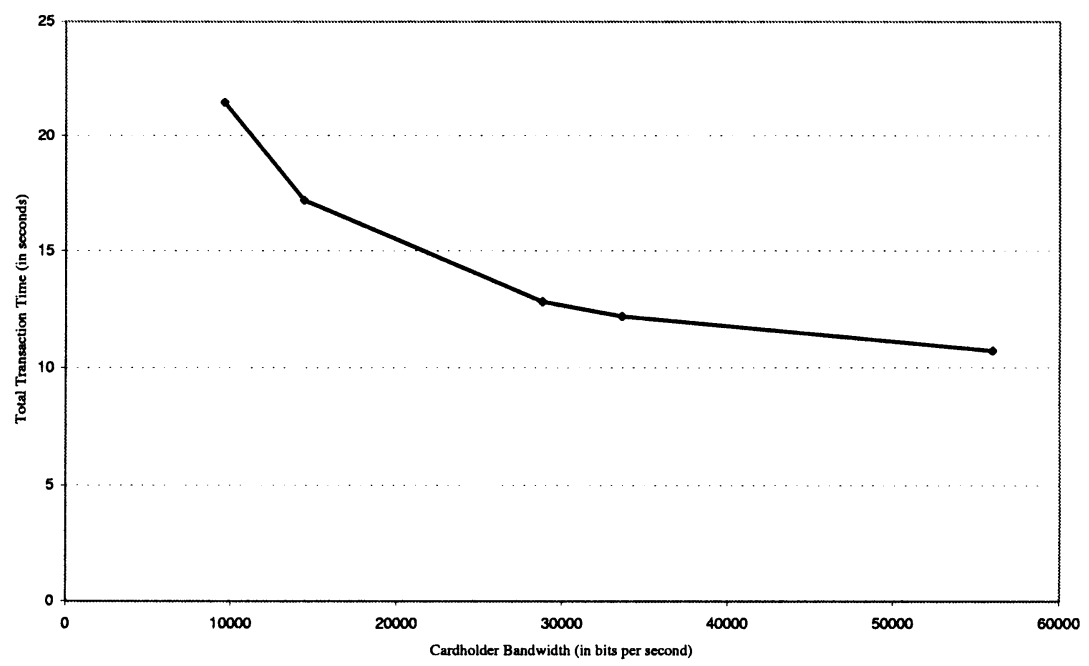

Figure 5 Graph of Transaction Time vs Cardholder Bandwidth. Merchant-Gateway link is $1 \mathrm{Mbps}$ and there is financial network delay of $100 \mathrm{~ms}$. 


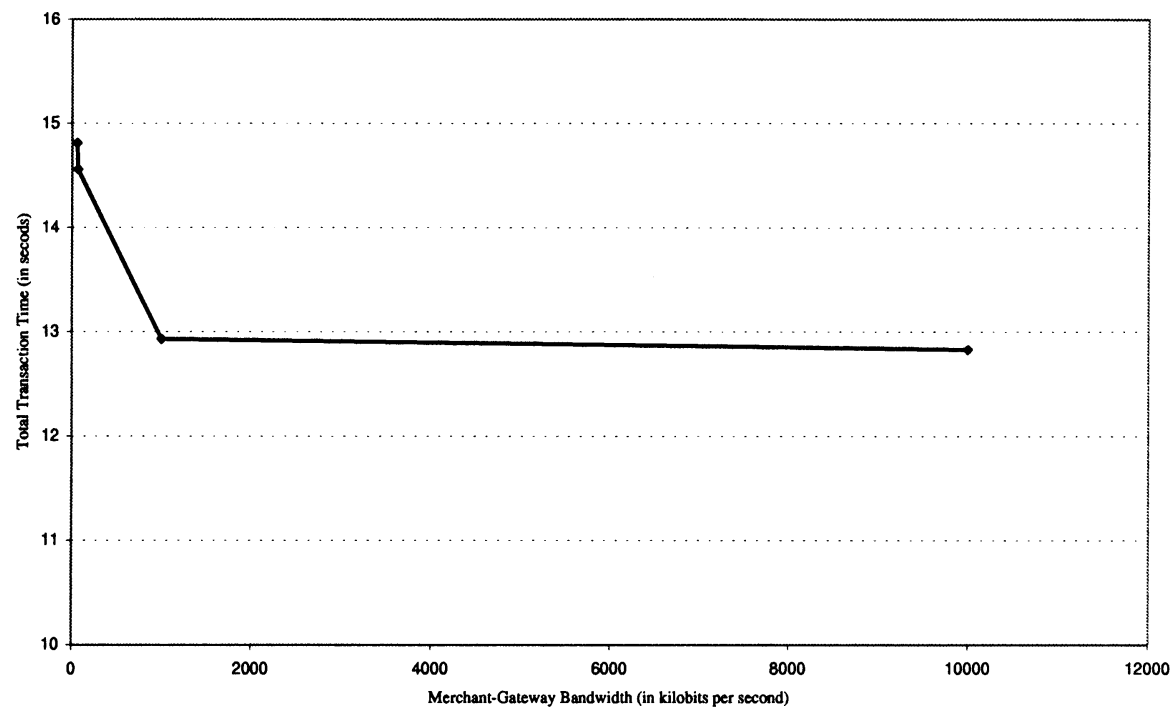

Figure 6 Transaction Times (in seconds) for Different Bandwidths and Latencies of the Merchant-Gateway link. The Cardholder-Merchant link is $28.8 \mathrm{Kbps}$ and the financial network delay is $100 \mathrm{~ms}$.

So far we have assumed that the gateway processes the authorization request at the rate measured from the implementation. This is not a valid assumption and the effect of a number of merchant's simultaneously requesting authorizations must be considered. The more simultaneous requests, the longer it will take to service the request. Thus by adjusting

\begin{tabular}{||c|c|c|c||}
\hline Bandwidth & \multicolumn{3}{|c||}{ Latency (ms) } \\
\cline { 2 - 4 } (bps) & 75 & 100 & 130 \\
\hline 9600 & 21.38 & 21.48 & 21.60 \\
\hline 14400 & 17.06 & 17.16 & 17.28 \\
\hline 28800 & 12.73 & 12.83 & 12.95 \\
\hline 33600 & 12.11 & 12.21 & 12.33 \\
\hline 56000 & 10.63 & 10.73 & 10.85 \\
\hline
\end{tabular}

Table 5 Transaction Times (in seconds) for Different Bandwidths and Latencies of the Cardholder-Merchant Link. The Merchant-Gateway link is $1 \mathrm{MBps}$ and the financial network delay is $100 \mathrm{~ms}$. 
the average service time of Gateway, this server's performance can be predicted.

The graph, Figure 8, shows the transaction time for $1,3,5,7$ and 9 simultaneous requests. Notice that not only does the transaction time increase, but the horizontal asymptote representing the upper bound for the cardholder request rate also shifts to the right. The gateway's performance thus affects the rate at which the merchant can service requests from the cardholder. The pie graph in Figure 9 shows the individual time components at an IAT $=100$ seconds for 9 simultaneous gateway requests.

These graphs illustrate the importance of the gateway processing time component. The effect of simultaneous requests at the gateway is only measured to 9 simultaneous requests. In reality this may be much greater and it is thus crucial to the performance of the system as a whole.

\section{CONCLUSION}

The following performance aspects of the SET purchase request were obtained from the model:

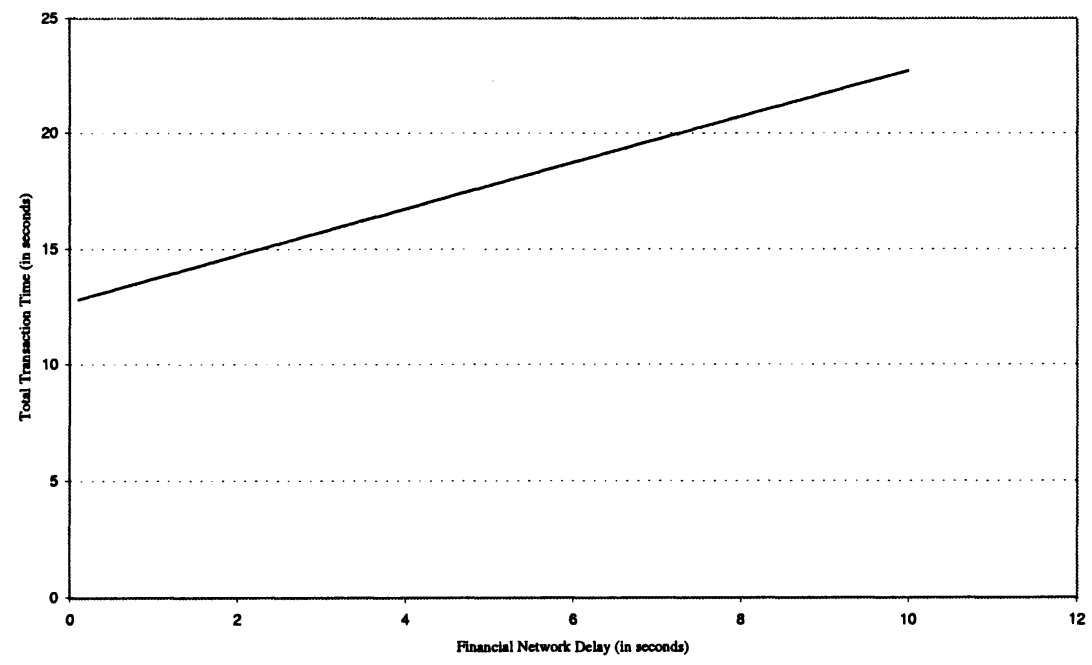

Figure 7 Graph of Transaction Time vs Financial Network Delay. The CardholderMerchant and Merchant-Gateway link capacities are kept constant at $28.8 \mathrm{Kbps}$ and $1 \mathrm{Mbps}$ respectively. 


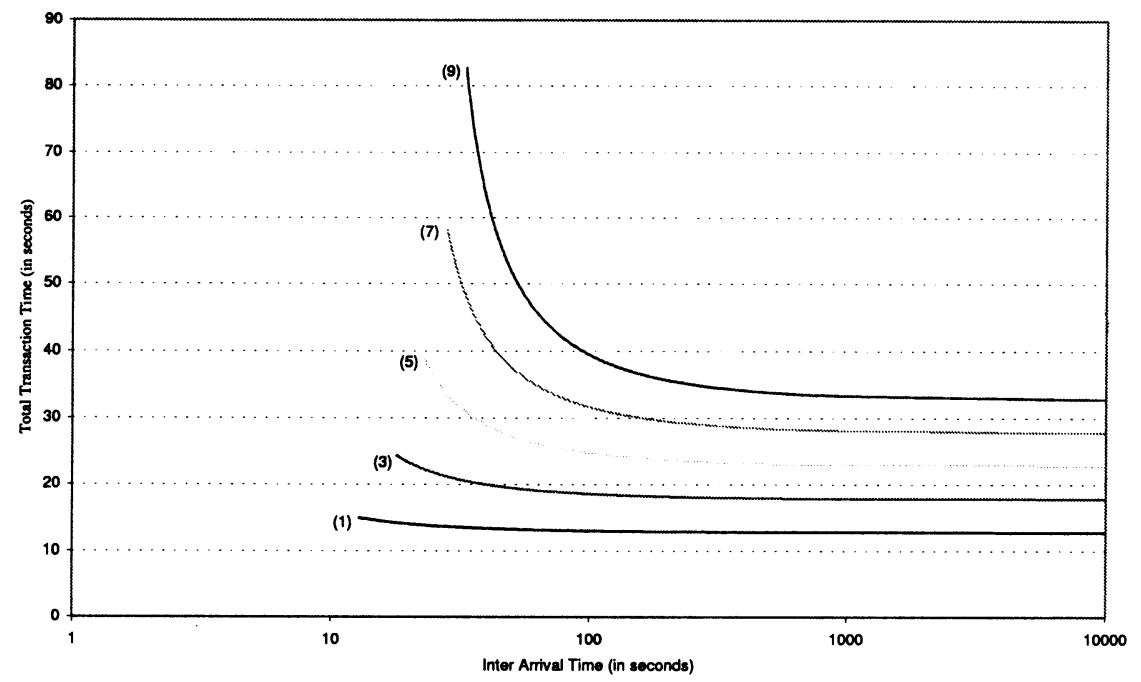

Figure 8 Graph of Transaction Time vs Interarrival Times for Different Gateway Server loads. The Cardholder-Merchant and Merchant-Gateway link capacities are kept constant at $28.8 \mathrm{Kbps}$ and $1 \mathrm{Mbps}$ respectively with a financial network delay of $100 \mathrm{~ms}$.

- The effect of the network is limited and existing infrastructures will suffice:

- Latency effects are negligible.

- The popular $28.8 \mathrm{Kbps}$ modem for the cardholder is sufficient.

- Common 1 Mbps merchant-gateway links are adequate.

- The financial network authorization delay directly affects the performance.

- Transaction times are strongly dependent on the merchant and especially the gateway processing component time.

The results obtained suggest that undertaking a performance modelling exercise on a transactional electronic commerce protocol is a valuable exercise. The general significance of modelling security protocols for performance is that assessment can be made as to their suitability for real world deployment.

In addition to the usefulness of the actual results obtained, the modelling approach that we have adopted could also be used to experiment 


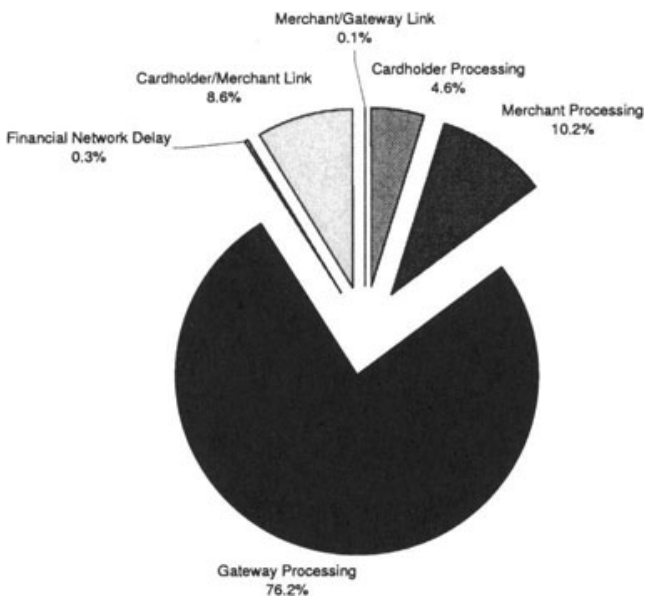

Figure 9 Individual Components of Transactional Time For Nine Simultaneous Requests for an IAT $=100$ seconds. The Cardholder-Merchant and Merchant-Gateway link capacities are kept constant at $28.8 \mathrm{Kbps}$ and $1 \mathrm{Mbps}$ respectively with a financial network delay of $100 \mathrm{~ms}$.

with the performance impact of changes to SET or in the implementation of new transactional electronic commerce protocols.

\section{Notes}

1. The individual cardholder, merchant and gateway implementations times were measured on a 170MHz Sun SPARCstation 5 workstation with 32MB memory, using Java JDK 1.1.6 and MySQL JDBC, and then averaged.

\section{References}

[1] SET Secure Electronic Transaction Specification. Book 1: Business Description. [http://www.setco.org/download/set_bk1.pdf], May 1997. Version 1.0.

[2] The Effects of Bandwidth and Latency on Internet-base Electronic Commerce. National Electronic Commerce Resource Center. Technology Development Activity. Johnstown, Pennysylvania, April 1997. [http://www.ecrc.ctc.com].

[3] Baskett, Chandy, Muntz and Palacios. Open, Closed and Mixed Networks of Queues with Different Classes of Customers. Journal of the ACM, 22(2):248-260, 1975. [http://www.cse.ucsd.edu/users/mihir/papers/oae.ps]. 
[4] M. Booyens and P.S. Kritzinger. SNAPL/1: A Language to Describe and Evaluate Queuing Network Models. Performance Evaluation, 4(3):171 - 181, August 1984.

[5] S. Cheshire. Bandwidth and latency: It's latency, stupid. TidBITS, (367), February 1997. [http://www.tidbits.com].

[6] Data Network Architectures Laboratory, University of Cape Town. MicroSnap - Multiclass Queuing Network Analyser (User Manual), third edition, August 1990.

[7] L. Gong. Lower Bounds on Messages and Rounds for Network Authentication Protocols. In Proceedings of the 1st ACM Conference on Computer and Communications Security, pages 26 - 37, Fairfax, Virginia, November 1993.

[8] L. Kleinrock. Queueing Systems, volume 1. John Wiley and Sons, 1975.

[9] M.S. Berry and E. Saul and A.C.M. Hutchison. jetSET - An Examination of the SET Protocol, 1998. Dissertation. 